\title{
The application of the space analytic geometry in the concrete example
}

\author{
Kanru Cheng ${ }^{1, \text { a }}$ \\ ${ }^{1}$ School of North China Electric Power University (Baoding), Baoding 071000, China; \\ a15176265865@163.com
}

Keywords: The inner cutting method, space analytic geometry, space linear fitting.

\begin{abstract}
This paper will aim at calculating parameters and solving its optimization problems of folding desk, according to the given plank and the size of the folding desk, deducing the calculation formula of the machining parameters making use of the geometrical relationship, establishing the multi-object programming mathematical model to calculate the optimum machining parameters, and provided several kinds of design scheme of the folding desk.
\end{abstract}

\section{Introduction}

One kind of the folding desk has circular desktop, desk's legs with activities of hinges can be amortized into a flat tablet. Legs consist of some battens. Divide them into two groups, each group of battens connected by rebar, both ends of the rebar are fixed to the two battens of the outermost table legs and there's some grooves along battens to ensure the freedom of sliding. Desk's surface composed of a ruled surface.

In this paper, we establish mathematical model aim at calculating parameters and solving its optimization problems of folding desk. The size of the rectangular plank given in the paper is $120 \mathrm{~cm}$ $\times 50 \mathrm{~cm} \times 3 \mathrm{~cm}$, each batten is $2.5 \mathrm{~cm}$ in wide, the rebar fastened to the center of the outermost batten of the legs. The height of folded desk is $53 \mathrm{~cm}$. We try to establish a model to describe the dynamic process of this folding desk, then we will have access to the designed and processing parameters of the folding desk(such as the length of the grooves on the batten of the desk's legs) and we also can describe the edge lines of table legs.

\section{Problem analysis}

Based on the material of the wooden plank and the size of the folding desk, establish the dynamic change model of folding desk and gives the design and processing parameter, that is the mathematic description of the length of the groove on the legs and the legs' edge line. According to the given size of the wooden planks and the battens can determine the number of the batten and the length of the wooden leg. Change the height of the desk, the tilt angle of each of the legs due to constraint of the rebar will change as well. Once the height of the desk was determined, the position of the rebar are also known, according to the spatial geometry and the knowledge of triangle, we can determine the tilt angle of the legs. The length of the groove on the legs and the space coordinates can be obtained by the tilt angle, then could get the mathematical description of the edge line according to the space curve fitting.

\section{Assumptions and symbol descriptions}

\subsection{Assumptions}

1. Assume the folding table's design all based on the ruled surface.

2. Plate material can guarantee a table does not break.

3. Legs' width and height were $2.5 \mathrm{~cm} \times 3 \mathrm{~cm}$ and will not change.

4. Ignore the impact of the rebar to the length of the groove. 


\subsection{Symbol descriptions}

Table 1 The symbol descriptions

\begin{tabular}{ccc}
\hline No. & symbol & Symbol Description \\
\hline 1 & $\mathrm{~B}$ & The wooden plank's width \\
2 & $\mathrm{~L}$ & The wooden plank's length \\
3 & $\mathrm{R}$ & The desktop's radius \\
4 & $\mathrm{Li}$ & The height of the leg i \\
5 & $\mathrm{li}$ & Half of the length of the batten i \\
6 & $\theta_{i}$ & The included angel of the batten i and the vertical direction \\
7 & ai & The distance between the innermost spot of the groove i and the hinge \\
8 & $\mathrm{bi}$ & The distance between the outermost spot of the groove i and the hinge \\
9 & $\mathrm{ci}$ & The length of the groove on the batten i \\
10 & $\mathrm{~h}$ & The height of the desk \\
11 & $\mathrm{~J}$ & The length of the gap between the two batten \\
12 & $\mathrm{e}$ & The distance between the two batten \\
\hline
\end{tabular}

\section{Model preparation}

\subsection{Simplified model processing}

The plank is a rectangle, the desktop is round, has the spatial symmetry. So the problem can transferred into dealing with the problem of a quarter of a round desktop and a quarter of a plank, as shown in Fig.1.

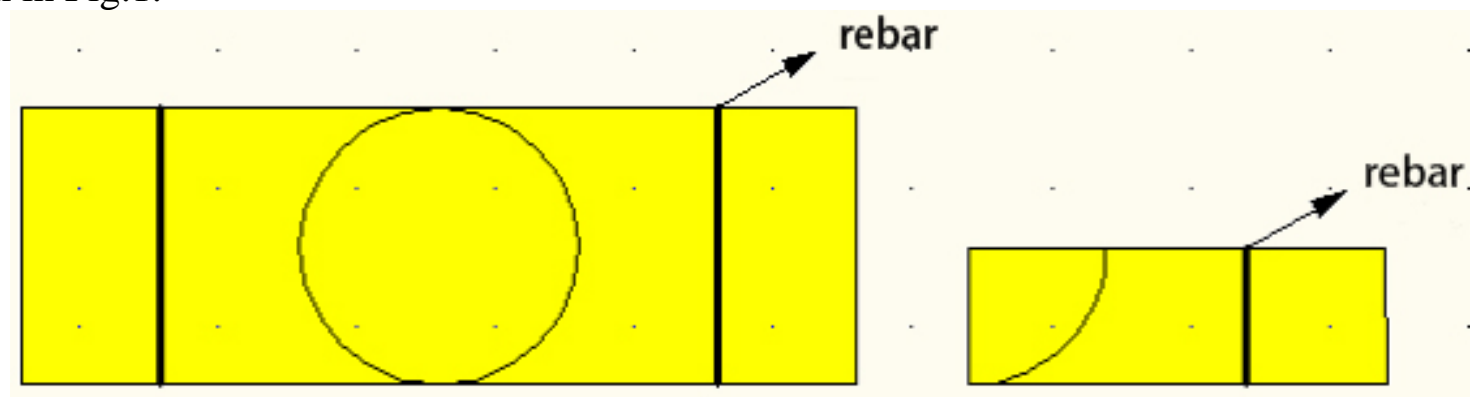

Fig.1 Simplified diagram

In order to simplify the expression of the article and make the figure more visualized and clear, transferred the three-dimensional figure into the two-dimensional figure, that is transforming the three-dimensional figure into plane geometry figure, as shown below.

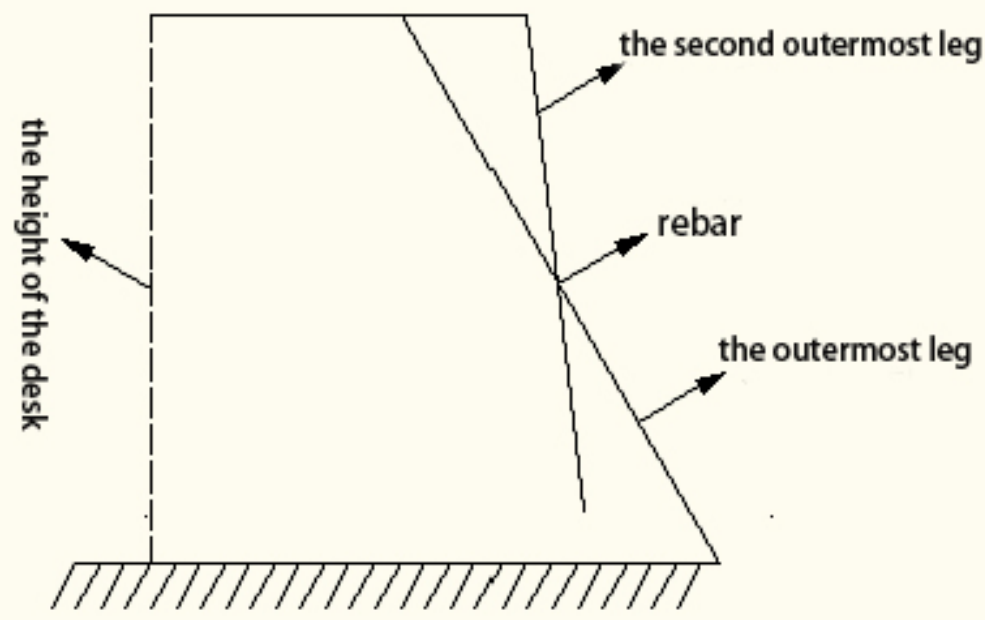

Fig. 2 The two-dimensional figure of the desk

The figure shows only the views of the outermost and the second outermost legs, other legs are the same. 


\subsection{The treatment to the thickness of the plank and the fold direction}

The thickness of the plate material itself contains certain. In the article, the height of the desktop considered to be the distance between the ground and the underside of the plank, the thickness of the desktop are not counted. The height of the desk is $53 \mathrm{~cm}$, the plank having a thickness of $3 \mathrm{~cm}$, when comes to the calculation of the height of the desk considered it to be $53 \mathrm{~cm}$.

The outer surface of the plank is a ruled surface. In general, ruled surface constitute by a family or several family of rectilinear [1], taking the characteristics of the ruled surfaces into account, the leg's folding direction is along the direction of the edge line of the plank. That is only considers legs folding direction along the length direction of the plank, other folding direction will not be discussed.

\subsection{The establishment of a three-dimensional rectangular coordinate system}

Folding the folding desk, when the outermost legs turn round, the rest of the legs turn round in traction of the rebar, each included angel of the leg and the vertical direction is changing constantly. For a more convenient description of folding the desk's dynamic changes, we have established a space rectangular coordinates, which origin to be the projection of the center of gravity of the desktop on the ground.

\section{Solve the problem}

Firstly, calculate the length of the batten on the desk and the length of desk's each leg. According to the given data, using two method, the inner cutting method and the external cutting method, calculate the length of the protruding batten. Secondly, build the dynamic change model when folded the folding desk. Using the included angle of the legs and the vertical plane, the length of the legs and the length of the battens on the desk descript the dynamic change quantitatively when folded the folding desk. Thirdly, the description of the legs' edge line. By calculating the spatial position of the legs under spatial coordinate system, fit the curvilinear parametric equation of legs' edge line, then draw the space curve. Fourthly, calculate the length of the empty groove of the legs. By calculating the distance between rebar and the position of the hinge on each leg under the condition of flat and fully expanded, the difference between this two distance is the length of the empty groove of each leg.

\subsection{The calculate of the length of the wood on the round desktop}

\section{The consideration of the gap between the batten}

The size of the plank is $120 \mathrm{~cm} \times 50 \mathrm{~cm} \times 3 \mathrm{~cm}$, each batten's width is $2.5 \mathrm{~cm}$, these battens is $2.5 \mathrm{~cm}$ in wide. In consideration of the friction would become larger, if divided the plank into 20 pieces, the resistance would become larger as well. Divided the plank into 19 pieces, there would has a certain gap between the battens $(J=0.1389 \mathrm{~cm})$.

$$
J=(50-2.5 \times 19) \div 18=0.1389 \mathrm{~cm}
$$

2. The calculation of the length of the protruding batten

(1) Two method

The round desktop of the folding desk was cut out from the plank, there are two method, the inner cutting method and the external cutting method, as shown in Fig.3 and Fig.4. 


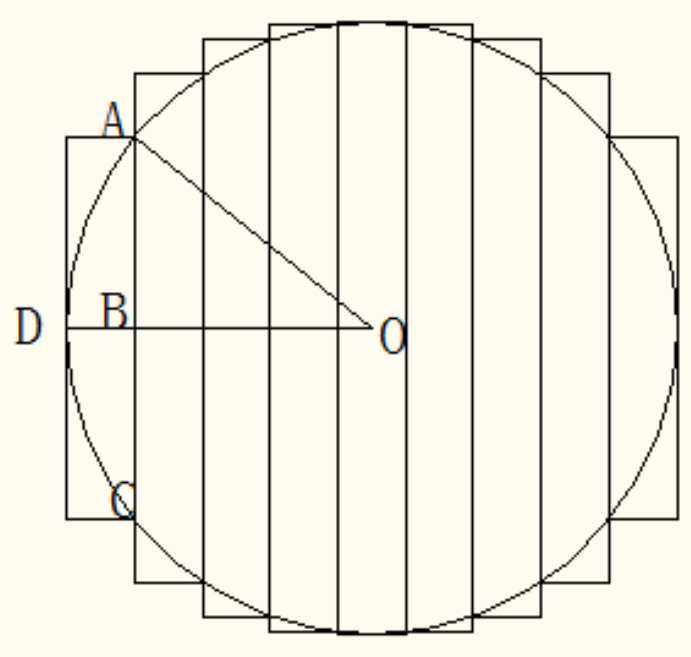

Fig.3 The inner cutting method

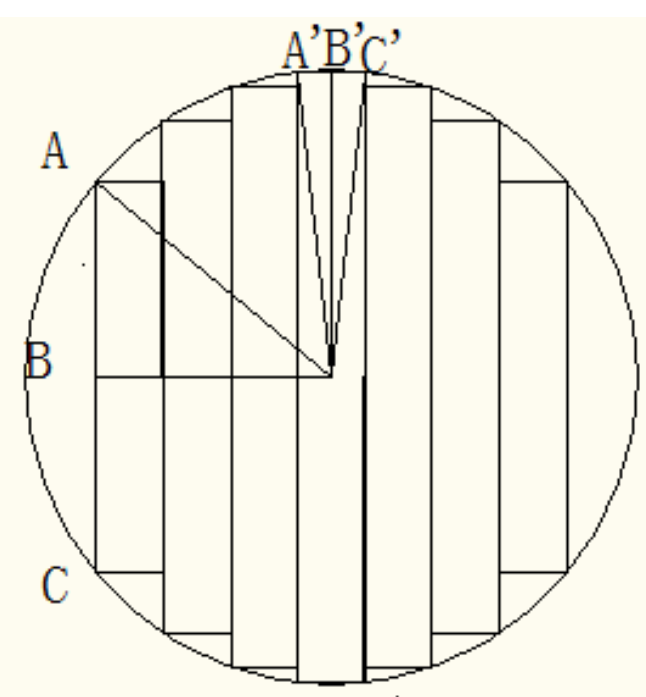

Fig.4 The external cutting method

Note: The gap between the planks is too narrow, so does not shown it here.

The battens are placed in order from left to right, and are numbered from 1 to 19 which are also the number of legs (a group of legs). The points $O$ of figures I and II are the center of the desktop.

As the figure III shows, according to external cutting method round desktop is tangent with battens 1 and 19. The points of intersection between the left of the desktop and battens 2 are A and C. Establishing perpendiculars of batten 1 respectively through the points $\mathrm{A}$ and $\mathrm{C}$. The perpendiculars are the cross section batten 1 and the position of the hinge between the desktop and NO.1 of desk's leg. Using desktop and batten 3 to establishing perpendicular of batten 2 and repeat this step until the batten 10. Each perpendicular is the cross section of every batten. Turn left from the right side of batten 19 to batten 10, which means that carrying out from both sides.

When using the inner cutting method, the round desktop and batten 1 will produce two different intersections $\mathrm{A}, \mathrm{C}$. Construction of the perpendicular of the $2^{\text {nd }}$ batten through point $\mathrm{A}$ and point $\mathrm{C}$ respectively, this perpendicular is the $1^{\text {st }}$ batten's cross-section. In the same way, construction of the perpendicular of the $3^{\text {rd }}$ batten through the two points of intersection of the $2^{\text {nd }}$ batten and the desktop, repeated this process until the $10^{\text {th }}$ batten. On the right side, repeated this process from the $19^{\text {th }}$ batten to the $10^{\text {th }}$ batten.

\section{(2) Project selection}

In order to judge the two different method of cutting the round desktop, we set constraints to cut off desktops according to the known conditions. The actual height of the fully extended rectangular plank is $50 \mathrm{~cm}$ and the length of the outermost desk's leg should satisfy the following inequality: $L_{1}>h=$ $50 \mathrm{~cm}$.

a) Calculate by the external cutting method

$O A=O C=25 \mathrm{~cm}, B D=2.5 \mathrm{~cm}, O B=O D-B D=22.5 \mathrm{~cm}$.

According to the Pythagorean theorem,

$$
A B=\sqrt{O A^{2}-O B^{2}}=10.9 \mathrm{~cm}
$$

$L_{1}$ is the half of the length of the plank minus $\mathrm{AB}$, that is

$$
L_{1}=\frac{L}{2}-A B=60-10.9=49.1<50=h
$$

It could not meet the condition.

b) Calculate by the inner cutting method 
$O A=O A^{\prime}=r, r$ is the radius of the round desktop, $O B=O B^{\prime}=25 \mathrm{~cm}$.

$\because \triangle \mathrm{OAB} \cong \triangle \mathrm{OA}^{\prime} \mathrm{B}^{\prime}$

$\therefore A B=A^{\prime} B^{\prime}=\frac{1}{2} \times 2.5=1.25 \mathrm{~cm}$

$$
L_{1}=\frac{L}{2}-A B=60-1.25=58.75>50=h
$$

It meets the condition, so choose the inner cutting method, the length of the protruding batten is $1.25 \mathrm{~cm}$.

3. The calculation of the length of the battens and the legs

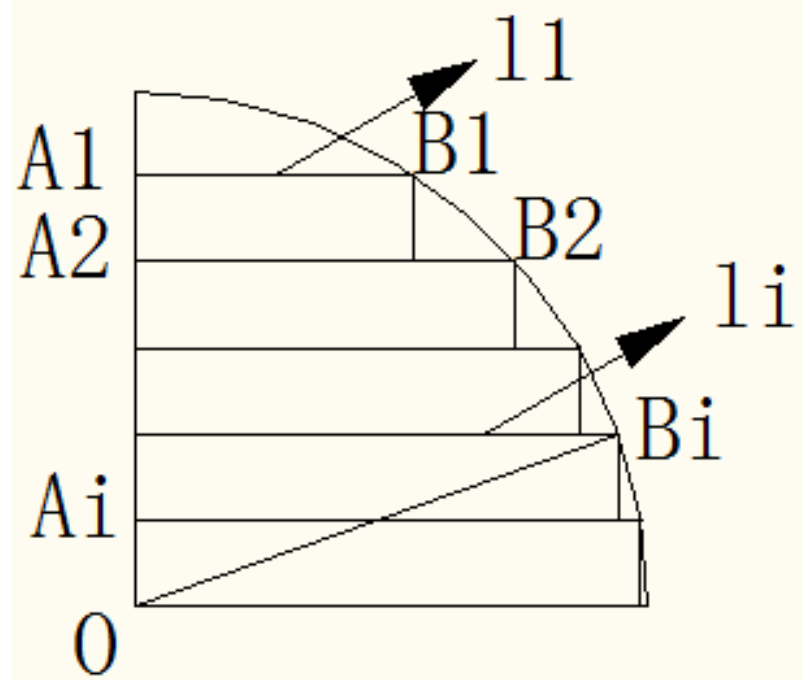

Fig.5 The figure of the desktop

According to Fig.5, $l_{1}=1.25 \mathrm{~cm}, O A_{i} A_{i+1}=e=(J+2.5) \mathrm{cm}$,

$$
\begin{gathered}
O A_{i}=O A_{1}-e(i-1) \\
O B_{i}=r=\sqrt{5^{2}-1.25^{2}}=25.03
\end{gathered}
$$

According to the Pythagorean theorem,

$$
l_{i}=A_{i} B_{i}=\sqrt{O B_{i}^{2}-O A_{i}^{2}}
$$

The length of the legs to be,

$$
L_{i}=L-l_{i}
$$

Using MATLAB calculates the length of the battens and the legs, the results are shown in Table 2.

Table 2 The length of the legs and the battens

\begin{tabular}{cccccc}
\hline NO. & 1 & 2 & 3 & 4 & 5 \\
\hline The length of the batten $(\mathrm{cm})$ & 1.250 & 11.249 & 15.414 & 18.295 & 20.443 \\
The length of the leg $(\mathrm{cm})$ & 58.750 & 48.751 & 44.586 & 41.705 & 39.557 \\
\hline NO. & 6 & 7 & 8 & 9 & 10 \\
\hline The length of the batten $(\mathrm{cm})$ & 22.072 & 23.292 & 24.165 & 24.727 & 25.000 \\
The length of the leg $(\mathrm{cm})$ & 37.928 & 36.708 & 35.835 & 35.273 & 35.000 \\
\hline
\end{tabular}

\subsection{To establish the dynamic change models about the folding process of folding table}

In the folding desk's folding process, when the outermost legs rotate, the rest of the leg is rotated by the rebar's traction, each included angel of the leg and the vertical direction is changing constantly. When the length of each leg, the included angle of the leg and the vertical direction and the position of the legs on the round desktop are determined, the spatial position of the legs can be determined. 
To facilitate the description of the dynamic process, we define the state vector of the legs to be $\left(l_{i}, L_{i}, \theta_{i}\right)$, by the quantitative analysis of the length of each desk's legs, the length of the legs' corresponding batten and the included angel, built a model of the parameter equation about the included angle of the leg and the vertical direction, determined these three parameter of each leg and realized the description of the dynamic changing process of folding desk.

1. The establishment of the model

Beginning with the outermost and the second outermost legs as the example of analysis, making the introductions by the figure of the batten below.

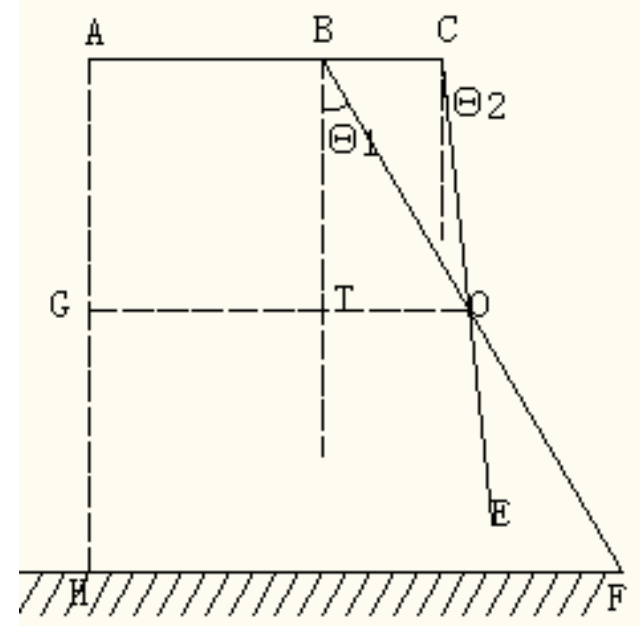

Fig.6 The figure of the plank

In the Fig.6, BF represents the outermost leg $\mathrm{L}_{1}$, $\mathrm{CE}$ represents the second outermost leg $\mathrm{L}_{2}$, point $\mathrm{O}$ represents the position of the rebar, $\theta 1$ represents the included angle of $\mathrm{BF}$ and vertical direction, $\theta 2$ represents the included angle of $\mathrm{CE}$ and vertical direction, $\mathrm{AC}$ represents the length of the batten on the round desktop which CE corresponded, AH represents $h$ which is the height of the desk.

According to the above, calculating the included angle of the leg and the vertical direction, $\mathrm{AB}=l_{1}, \mathrm{AC}=l_{2}, \mathrm{BO}=\frac{1}{2} B F=\frac{L_{1}}{2}, \mathrm{BT}=\mathrm{AG}=\frac{h}{2}$

According to $\triangle \mathrm{BTO}$

$$
\begin{aligned}
& \cos \theta_{1}=\frac{B T}{B O}=\frac{h}{L_{1}} \\
& \theta_{1}=\arccos \frac{h}{L_{1}}
\end{aligned}
$$

According to $\mathrm{GT}+\mathrm{TO}=\mathrm{GD}+\mathrm{DO}$

$$
T O=h \tan \theta_{1}, D O=h \tan \theta_{2}
$$

$\because G T=A B=l 1, G D=A C=l 2$

$$
\begin{aligned}
& l_{1}+\frac{h}{2} \tan \theta_{1}=l_{2}+\frac{h}{2} \tan \theta_{2} \\
& \theta_{2}=\arctan \frac{2 l_{1}-2 l_{2}+h \tan \theta_{1}}{h}
\end{aligned}
$$

In the same way

$$
\theta_{i}=\arctan \frac{2 l_{1}-2 l_{i}+h \tan \theta_{1}}{h}(i=1,2, \cdots, 10)
$$

2. The solution of the model 
Solving the problem by using MATLAB, combined the results of the solution in 4.1 about the length of batten and the length of the legs and list $\left(l_{i}, L_{i}, \theta_{i}\right)$ these three parameters in the one table. Shown in the following table (Corresponding to the greatest degree of folding table leg at this time).

Table 3 The state vector of the batten

\begin{tabular}{ccccccc}
\hline NO. & 1 & 2 & 3 & 4 & 5 \\
\hline The state vector of the batten & $\left(l_{i}, L_{i}, \theta_{i}\right)$ & $(1.25$, & $(11.25$, & $(15.41$, & $(18.30$, & $(20.44$, \\
& 58.75, & 48.75, & 44.59, & 41.70, & 39.56, \\
& $31.67)$ & $12.24)$ & $2.88)$ & $-3.71)$ & $-8.57)$ \\
\hline NO. & 6 & 7 & 8 & 9 & 10 \\
\hline The state vector of the batten & $\left(l_{i}, L_{i}, \theta_{i}\right)$ & $(23.29$, & $(24.17$, & $(24.73$, & $(25.00$, & $(23.29$, \\
& & 36.71, & 35.83, & 35.27, & 35.00, & 36.71, \\
& $-14.83)$ & $-16.68)$ & $-17.86)$ & $-18.42)$ & $-14.83)$ \\
\hline
\end{tabular}

Note: The unit of $\theta_{i}$ is degree

This chart included the data about the legs which corresponding to a quarter circle. In other words, the outcome is about the length of each desk's legs, the length of the legs' corresponding batten and the included angel when folded the most. The negative angle refers to deflection angle of the table leg, which is greater than ninety degrees.

While the $\theta_{i}$ of the outermost leg changing, each leg's $\theta_{i}$ changes as well. To be specific, the leg's $L_{i}$ has a corresponding $\theta_{i}$ if it is given a specific $\theta_{i}$. Because the state vector $\left(l_{i}, L_{i}, \theta_{i}\right)$ of each table leg will change when $\theta_{1}$ is changing, the descriptions of the leg's folding process might through the state vector $\left(l_{i}, L_{i}, \theta_{i}\right)$ of each leg.

\subsection{Solving and analyzing the legs' edge line quantitatively}

In order to make the mathematical description of the parameters of the legs' edge line, we might achieve it by fitting the position of the legs' edge line in a space coordinates system and then draw a space curve.

\section{Calculation of the curve parametric equations}

Establishing a space rectangular coordinates system, which origin is the projection of the center of gravity of the desktop on the ground. Then combine the vector parameter calculation of the folding desk in $\mathbf{4 . 2}$ and shown the position of each leg use the coordinate.

The formula to be

$$
\left\{\begin{array}{c}
x_{i}=\frac{B}{2}-1.25-(2.5+J)(i-1) \\
y_{i}=l_{i}+L_{i} \sin \theta_{i} \\
z_{i}=h-L_{i} \cos \theta_{i} \\
i=1,2, \cdots, 10
\end{array}\right.
$$

$B$ represents the width of the plank $(B=50 \mathrm{~cm}$ ), $J$ represents the gap between each batten, $i$ represents the number of the batten, $L_{i}$ represents the length of desk's legs, $\theta_{i}$ represents the included angel of the leg and the vertical direction, $h$ represents the height of the table, $l_{i}$ represents the length of the batten. The parameters have been calculated, substituting into the above equation to determine the spatial position of the legs and then might draw the legs' edge line. Furthermore, use the least squares method fitting the parametric equation curve of the legs' edge line.

2. The results

Using MATLAB calculates the spatial coordinates of the legs. 
Table 4 The spatial coordinates of the legs

\begin{tabular}{cccccc}
\hline NO. & 1 & 2 & 3 & 4 & 5 \\
\hline The legs' coordinates $(x, y, z)$ & $(23.75$, & $(21.11$, & $(18.47$, & $(15.83$, & $(13.19$, \\
& $32.10,0.00)$ & $21.59,2.36)$ & $17.66,5.47)$ & $15.60,8.38)$ & 14.55, \\
& & & & & $10.89)$ \\
\hline NO. & 6 & 7 & 8 & 9 & 10 \\
\hline The legs' coordinates $(x, y, z)$ & $(10.56$, & $(7.92$, & $(5.28$, & $(2.64$, & $(0.00$, \\
& 14.07, & 12.90, & 13.88, & 13.91, & 13.94, \\
& $12.93)$ & $14.51)$ & $15.67)$ & $16.43)$ & $16.79)$ \\
\hline
\end{tabular}

We might obtain a space curve and three projected curves which are on three planes, if we use curve fitting into spatial coordinates.

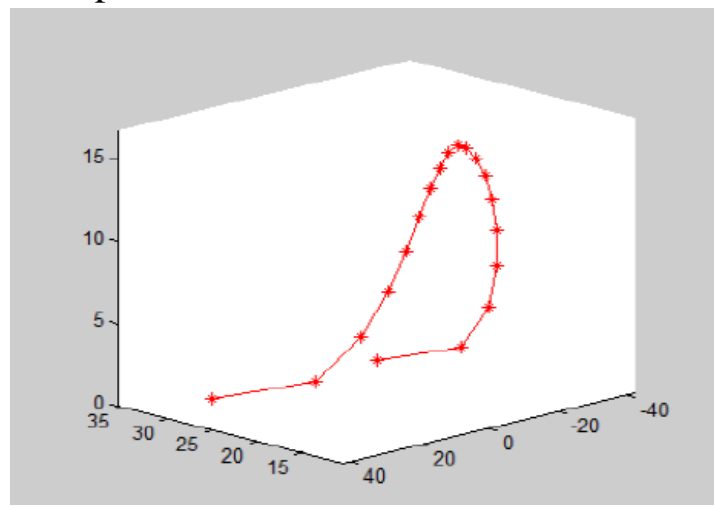

Fig.7 The space curve

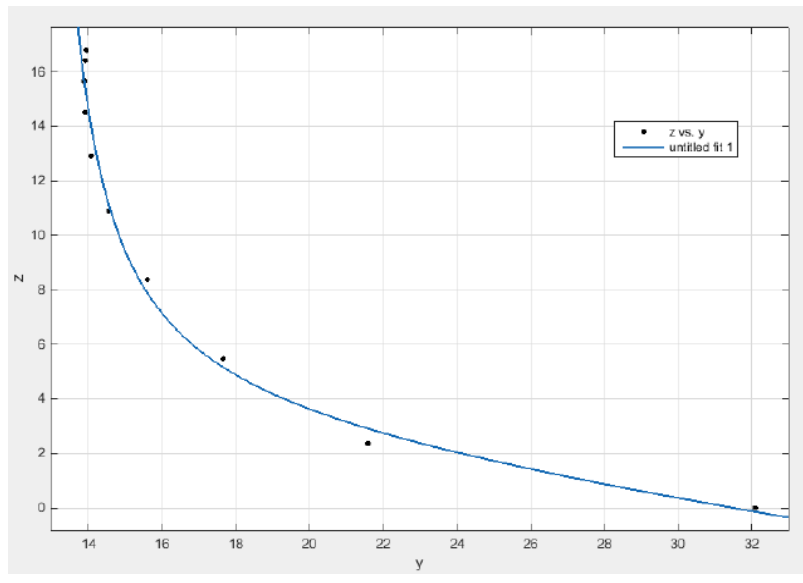

Fig.9 YZ planar projection curve

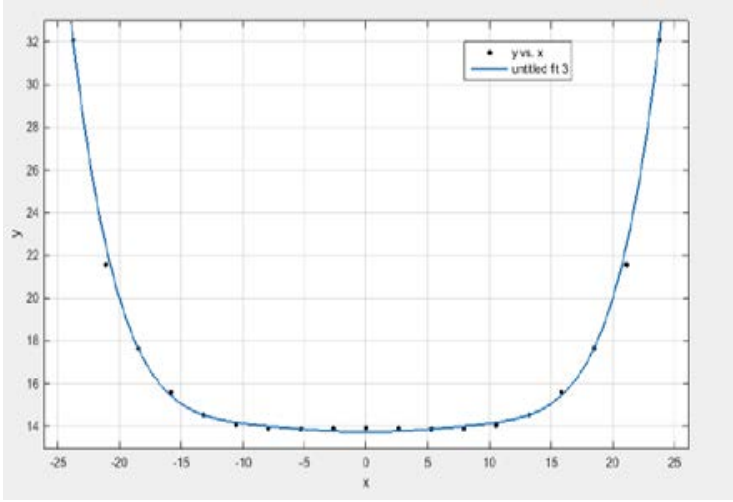

Fig.8 XY planar projection curve

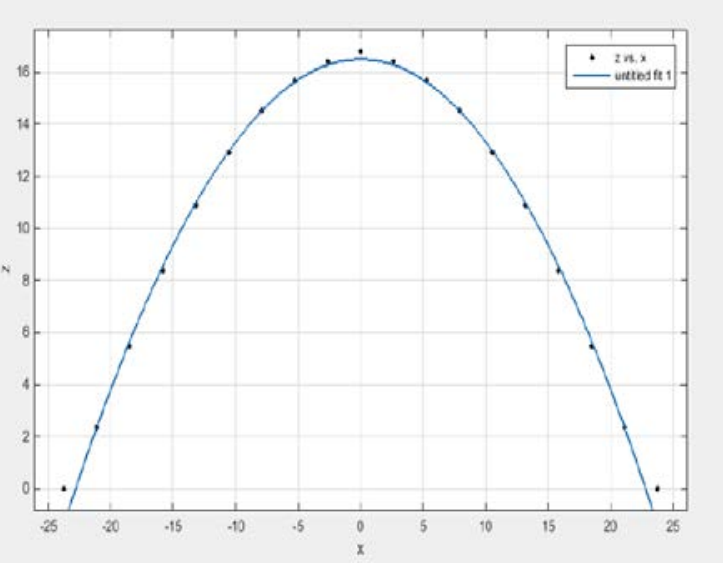

Fig.10 XZ planar projection curve

\subsection{The calculation of the length of each groove on desk's legs}

\section{The process of calculation}

The desk's outermost leg would move when folding the folding desk, the rebar on other legs would move in the groove, the rebar's movement was shown in Fig.11. 


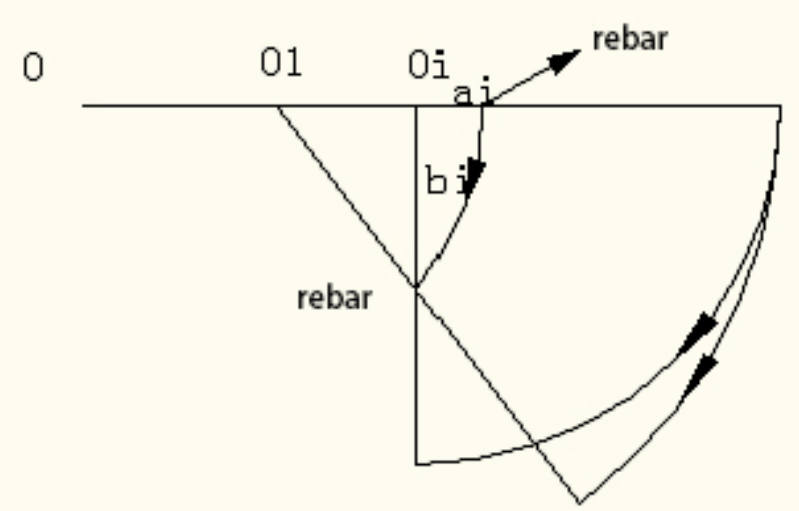

Fig.11 The figure of the movement of the rebar

Combining with 4.2 and analyzing Fig.11,

$$
\begin{gathered}
a_{i}=l_{1}+\frac{L_{1}}{2}-l_{i} \\
b_{i}=\frac{h}{2 \cos \theta_{i}}
\end{gathered}
$$

The length of the groove is

$$
c_{i}=b_{i}-a_{i}(i=2,3, \cdots, 10)
$$

$a_{i}$ represents the distance between the joint of the desk's leg and the desktop and rebar when unfolding the folding desk, $b_{i}$ represents the distance between the joint of the desk's leg and the desktop and rebar when folding the folding desk. $L_{1}$ represents the length of the outermost leg, $l_{i}$ represents the length of the batten, $\theta_{i}$ represents the included angel of the leg and the vertical direction.

2. The result of calculation

Table 5 The length of the groove

\begin{tabular}{cccccc}
\hline NO. & 1 & 2 & 3 & 4 & 5 \\
\hline The length of the groove $(\mathrm{cm})$ & 0.000 & 6.206 & 9.821 & 12.723 & 15.101 \\
\hline NO. & 6 & 7 & 8 & 9 & 10 \\
\hline The length of the groove $(\mathrm{cm})$ & 17.024 & 18.529 & 19.638 & 20.367 & 20.725 \\
\hline
\end{tabular}

According to the result, the length of the groove of the middlemost batten is longest, the outermost batten might not has the groove, the length of the groove of each batten might not according to the distribution of arithmetic progression. The more the batten near the middle section, the more their length are closed to each other. In addition, the length of the groove is in a symmetrical distribution, it means the length of the groove of the $11^{\text {th }}$ batten is equal to the length of the groove of the $9^{\text {th }}$ batten, the summation of the length of the batten is $259.54 \mathrm{~cm}$.

\section{References}

[1] Cai, G.L., Li, Y.X.,\&Wang, S.H.(2008). Property of Ruled Surface and its application in engineering. Mathematics in Practice and Theory,38(8), 98-102.

[2] Lin, M.,Dong, Y.C.,\&Liu, H.Y.(2010). Some Applications of MATLAB in the Teaching of Space Analytic Geometry. Time Education, 4, 98-100.

[3] Cheng, G.,\&Li, C.(2015). The Application of Matlab in Space Analytical Geometry Teaching. Microcomputer Applications, 31(6), 45-51.

[4] Cui, Q.Z.(2012). Design and Implementation of Matlab for Quadric Surface Expansion and Contraction Method in Space Analytic Geometry. Computer \& Telecommunication, 8, 45-50.

[5]Kong, X.Q.(2012). Exploring the application of Matlab software in space analytical geometry teaching. Computer Applications and Software, 29(8), 297-300.

[6] Yang, W.M.(2004). Space Analytical Geometry. Wuhan, MA: Wuhan University Press. 